\title{
Clinicoradiopathological Features among Mediastinal Masses
}

\author{
R. Sridhar, R. Narasimhan, L. Sundararajan, Raj B. Singh \\ Department of Respiratory Medicine, Apollo Main Hospital, Chennai, Tamil Nadu, India
}

\section{Abstract}

Background: The mediastinum is a place where various benign and malignant diseases usually manifest as mass and present as interesting diagnostic challenge. The purpose of this study was to describe clinical features, radiological, and pathological information of mediastinal masses to help have an organized approach to diagnosis of mediastinal masses. Patients and Methods: This was a prospective, descriptive cross-sectional study conducted over a period of 1 year at our hospital after obtaining ethical committee clearance. Patients with mediastinal masses fulfilling the inclusion criteria were enrolled in the study. Patient's clinical history, radiological features, techniques used to obtain specimens, and cyto-histopathology results from data collected in our total study population were analyzed. Results: A total of 73 patients with mediastinal masses were included. Thirty-three of them (45.2\%) were malignant and 32 (43.8\%) were nonmalignant masses. The masses were commonly located in the middle compartment $(n=42[57.5 \%])$, followed by anterior compartment (20 [27.4\%]), posterior compartment (8 [11\%]), and multicompartment (3 [4.1\%]). Among middle mediastinal masses, infectious masses were $14(33.3 \%)$, followed by 11 (26.2\%) malignant masses. Anterior mediastinal masses were predominantly malignant in nature (90\%). Nature of mass was inconclusive in eight (11\%) patients. Conclusion: Clinical history, anatomical position, and imaging characteristics allow the correct diagnosis in many cases when it is combined with histopathology. The newer endoscopic techniques such as endoscopic ultrasound-guided fine-needle aspiration or biopsy are generally preferred in view of lesser complications and ease compared to more invasive surgical procedures for mediastinal mass evaluation.

Keywords: Endobronchial ultrasound, endoscopic ultrasound, mediastinal lymphadenopathy, mediastinal mass

\section{INTRODUCTION}

The mediastinum is the central part of thoracic cavity which is bounded by pleural sacs, sternum, and vertebral bodies ${ }^{[1]}$ It contains various structures such as heart, thymus, lymph nodes, major blood vessels, nerves, trachea, and esophagus. Various benign and malignant diseases manifest as mediastinal mass and pose an interesting diagnostic challenge. ${ }^{[2]}$

Etiology of mediastinal mass varies with age and gender. These masses can present with multiple symptoms depending on many factors such as age, location, compression of adjacent structures, and nature of the disease. One-third of patients will be asymptomatic. ${ }^{[3-5]}$

Mediastinal masses are most often detected by chest X-ray. However, small masses due to mediastinal adenopathy can be detected only by computed tomography (CT). Multidetector CT can show more characteristic features of mediastinal mass and can be helpful in planning further workup of mediastinal mass as well as narrow down the differential diagnosis.

\begin{tabular}{|l|l|}
\hline \multicolumn{2}{|c|}{ Access this article online } \\
\hline Quick Response Code: & Website: \\
\hline & www.ijrc.in \\
\cline { 2 - 2 } & \\
\hline
\end{tabular}

Various classification methods such as Shields, Felson, Heitzman, Frazer, Zylak, and Whitten methods were used for division of mediastinum, and there is no uniqueness in classification across the world. ${ }^{[6-11]}$ Even though diagnosis can be arrived with the help of clinical and radiological features, tissue diagnosis is often required to guide the management of mediastinal masses. ${ }^{[12-14]}$

The objective of this study was to describe the clinical features, radiological features, and cytology or histopathological features of mediastinal masses.

\section{Patients and Methods}

This descriptive cross-sectional study included all patients with mediastinal mass who underwent biopsy or aspiration

Address for correspondence: Dr. R. Sridhar, Department of Respiratory Medicine, Apollo Main Hospitals, Greams Road, Chennai, Tamil Nadu, India. E-mail: dr.sridhar.ily@gmail.com

This is an open access journal, and articles are distributed under the terms of the Creative Commons Attribution-NonCommercial-ShareAlike 4.0 License, which allows others to remix, tweak, and build upon the work non-commercially, as long as appropriate credit is given and the new creations are licensed under the identical terms.

For reprints contact: WKHLRPMedknow_reprints@wolterskluwer.com

How to cite this article: Sridhar R, Narasimhan R, Sundararajan L, Singh RB. Clinicoradiopathological features among mediastinal masses. Indian J Respir Care 2021;10:41-6.

Received: $21-05-2020$

Accepted: $12-09-2020$
Revised: 03-09-2020 Published: $31-01-2021$ 
in our study setting during the study period from November 2017 to December 2018. The study commenced after obtaining institutional ethics committee clearance. After obtained informed consent, 76 patients were enrolled in the study.

Patient's demographic details such as gender, age, body mass index, occupation, and smoking were obtained. Detailed clinical history regarding cardinal respiratory symptoms, constitutional symptoms, and compression symptoms such as hoarseness, dysphagia, dyspnea, and swelling of the face and systemic syndromes such as myasthenia gravis and thyroid dysfunction which are likely to be associated with mediastinal masses were obtained from patients undergoing evaluation.

Radiological features of mediastinal masses recorded included mediastinal widening in the chest X-ray and location of mediastinal mass in CT scan. Various invasive techniques used to obtain cytology or biopsy specimens from mediastinal mass such as image-guided percutaneous biopsy or fine-needle aspiration (FNA), endobronchial ultrasound-guided transbronchial needle aspiration (EBUS-TBNA), endoscopic ultrasound-guided fine-needle aspiration (EUS-FNA), video-assisted thoracoscopic surgery (VATS), and surgical excision by sternotomy or thoracotomy were also noted. Pathology reports of tissue specimens either cytology or biopsy obtained from mediastinal mass were collected. Demographic details, clinical history, radiological features, techniques used to obtain specimens, and cyto-histopathology results from data collected in our total study population were entered in MS Excel (version 2007) and analyzed using SPSS software (IBM Corp. Released 2012. IBM SPSS Statistics for Windows, Version 21.0. Armonk, NY: IBM Corp).

\section{RESULTS}

In this study, 73 patients with mediastinal masses were included in the study. The mean age of the patients was 44.7 years (range 16-75 years) and more prevalent in the age range of 30-50 years. Forty-four patients $(60.3 \%)$ were male with a male-to-female ratio of $3: 2$. Only twenty $(27.3 \%)$ patients were smokers [Table 1]. The presenting symptoms, compressive symptoms, and constitutional symptoms and their frequencies are listed in Table 1. Among the twenty patients with anterior mediastinal mass, seven patients had systemic syndromes of whom, myasthenia gravis was present in five $(25 \%)$ patients, and they were diagnosed to have thymoma [Table 1]. This association of systemic syndromes with location was also significant $(P<0.001)$.

Anterior mediastinal masses were more common in patients $<30$ years, whereas middle mediastinal masses were common in patients above 30 years [Figure 1]. Breathlessness and cough were the most common symptoms followed by chest pain. Compressive symptoms were absent in $80 \%$ of the patients. The relationship of compressive symptoms to location was found to be very highly significant $(P=0.000)$. Back pain was the only compressive symptom in four of the eight patients who had posterior mediastinal tumors. Five out of the twenty patients with anterior mediastinal mass

\begin{tabular}{|c|c|}
\hline Clinical parameters & $n(\%)$ \\
\hline \multicolumn{2}{|l|}{ Gender } \\
\hline Male & $44(60.3)$ \\
\hline Female & $29(39.7)$ \\
\hline Age in years, mean (range) & $44.7(16-75)$ \\
\hline \multicolumn{2}{|l|}{ Smoking } \\
\hline Nonsmoker & $53(72.6)$ \\
\hline Smoker & $20(27.4)$ \\
\hline \multicolumn{2}{|l|}{ Symptoms } \\
\hline Asymptomatic & $5(7)$ \\
\hline Cough & $31(42.5)$ \\
\hline Sputum & $4(5.5)$ \\
\hline Wheeze & $4(5.5)$ \\
\hline Hemoptysis & $4(5.5)$ \\
\hline Chest pain & $15(20.5)$ \\
\hline Breathlessness & $36(49.3)$ \\
\hline \multicolumn{2}{|l|}{ Compressive symptoms } \\
\hline None & $59(80.8)$ \\
\hline Hoarseness of voice & $4(5.5)$ \\
\hline Difficulty in swallowing & $2(2.7)$ \\
\hline Back pain & $4(5.5)$ \\
\hline Both hoarseness of voice and difficulty in swallowing & $3(4.1)$ \\
\hline Facial puffiness & $1(1.4)$ \\
\hline \multicolumn{2}{|l|}{ Constitutional symptoms } \\
\hline None & $39(53.4)$ \\
\hline Fever & $10(13.7)$ \\
\hline Loss of weight & $10(13.7)$ \\
\hline Loss of weight, fever, and loss of appetite & $6(8.2)$ \\
\hline Fever and loss of weight & $6(8.2)$ \\
\hline Loss of weight and loss of appetite & $2(2.7)$ \\
\hline \multicolumn{2}{|l|}{ Systemic syndromes } \\
\hline No & $64(87.7)$ \\
\hline Myasthenia gravis & $5(6.8)$ \\
\hline Hypothyroid & $3(4.1)$ \\
\hline Hyperthyroid & $1(1.4)$ \\
\hline \multicolumn{2}{|l|}{ Imaging: Location of mass } \\
\hline$>1$ compartment & $3(4.1)$ \\
\hline Anterior & $20(27.4)$ \\
\hline Middle & $42(57.5)$ \\
\hline Posterior & $8(11)$ \\
\hline
\end{tabular}

had compressive symptoms such as hoarseness of voice and difficulty in swallowing and facial puffiness. Only three patients of the 42 patients with middle mediastinal mass had compressive symptoms, either hoarseness of voice or difficulty in swallowing [Figure 2].

Among the mediastinal masses, 33 (45.2\%) were malignant and $32(43.8 \%)$ were nonmalignant. Tuberculous mediastinal lymphadenitis was the diagnosis in $15(20.5 \%)$ patients, followed by thymoma and sarcoidosis in $11(15.1 \%)$ patients each and lung carcinoma in $10(13.7 \%)$ patients. Table 2 shows the analysis of the final diagnosis and nature of mediastinal masses.

The chest radiograph showed evidence of mediastinal mass in $78.8 \%$ of malignant masses. The chest radiograph showed 
Sridhar, et al.: Features of mediastinal masses

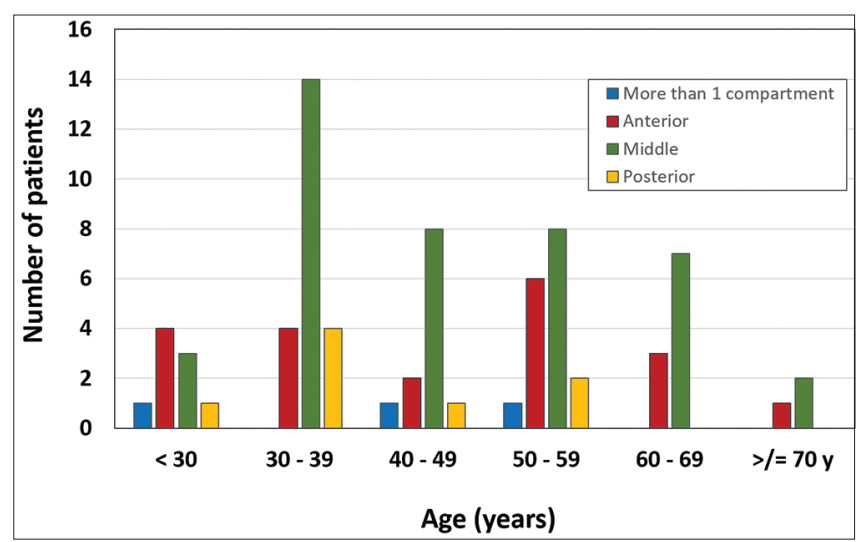

Figure 1: Distribution of location of masses with age

evidence of mediastinal mass in all three (100\%) patients with multiple compartment mass, 19 (95\%) patients with anterior mediastinal mass, and $8(19 \%)$ patients with a middle mediastinal mass.

Lesion was most common in middle compartment (42 [57.5\%]), followed by anterior compartment (20 [27.4\%]), posterior compartment (8 [11\%]), and multicompartment (3 [4.1\%]). Anterior mediastinal masses were predominantly malignant in nature $(90.0 \%)$. Infectious masses $(33.3 \%)$ were more common among the middle mediastinal masses followed by malignancy $(26.2 \%)$. Two of the three multiple compartment masses were malignant. Half of the posterior mediastinal masses were noninfectious and nonmalignant. Nature of mass was predominantly inconclusive in $16.7 \%{ }^{[7]}$ of middle mediastinal masses. Table 2 shows the distribution of the final diagnosis of mediastinal masses among mediastinal compartment.

EBUS, EUS, and VATS were performed only in middle mediastinal masses in this study population. CT-guided biopsy/ FNAC was done in $50 \%{ }^{[10]}$ of anterior mediastinal masses and posterior mediastinal masses. Surgery was done in the rest of the anterior and posterior mediastinal masses. Table 3 shows the analysis of techniques used to obtain specimens among the location of masses. Malignant masses were diagnosed by CT-guided biopsy followed by surgery in 30.3\% ${ }^{[10]}$ Each EBUS and EUS techniques were used in $31.3 \%$ of infectious masses. The final diagnoses were arrived in 65 out of $73(89 \%)$ patients. Among the 73 patients in our study, 33 (45.2\%) were malignant, 16 (21.9\%) infectious, 16 (21.9\%) noninfectious, and inconclusive in $8(11 \%)$ patients.

There was a significant association $(P=0)$ of the technique used to obtain specimens for analysis and the location of the mass [Table 4]. Ten of the total twenty patients with anterior mediastinal mass had a CT-guided biopsy, while the rest underwent surgery. EBUS, EUS, and VATS were performed only in middle mediastinal masses in this study group. CT-guided biopsy/FNAC was done in four (50\%) out of eight patients with posterior mediastinal masses, while surgery was done in the rest. The final diagnoses were arrived in 65 out of $73(89 \%)$ patients.

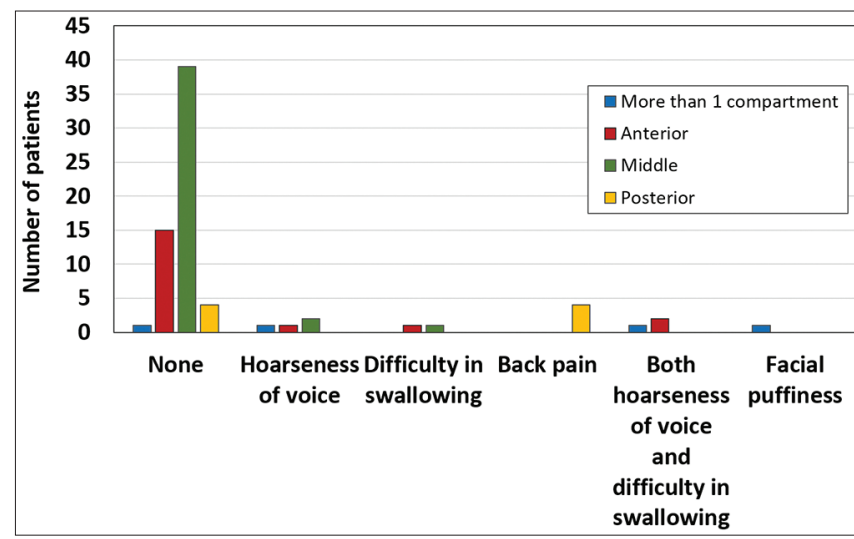

Figure 2: Description of compressive symptoms and the location of masses Table 2: Analysis of final diagnosis and nature of
mediastinal masses

\begin{tabular}{lc}
\hline Final diagnosis & $\boldsymbol{n}(\%)$ \\
\hline Malignant & $33(45.21)$ \\
Thymoma & $11(15.1)$ \\
Lung cancer & $10(13.7)$ \\
Neuroendocrine tumor & $4(5.5)$ \\
Lymphoma & $2(2.7)$ \\
Mature cystic teratoma & $1(1.4)$ \\
Retrosternal goiter (papillary cell carcinoma) & $1(1.4)$ \\
Solitary fibroid tumor & $1(1.4)$ \\
Metastasis & $2(2.7)$ \\
Poorly differentiated carcinoma & $1(1.4)$ \\
Nonmalignant & $32(43.8)$ \\
Noninfectious & $16(21.9)$ \\
Castleman's disease & $1(1.4)$ \\
Schwannoma & $2(2.7)$ \\
Bronchial cyst & $1(1.4)$ \\
Thymolipoma & $1(1.4)$ \\
Granulomatous lymphadenitis - sarcoidosis & $11(15.1)$ \\
Infectious & $16(21.9)$ \\
Granulomatous lymphadenitis - tuberculosis & $15(20.5)$ \\
Tuberculous paraspinal abscess & $1(1.4)$ \\
Inconclusive & $8(11)$ \\
Total & $73(100)$ \\
\hline &
\end{tabular}

\section{Discussion}

Mediastinal masses are a diverse group of lesions, and they continue to represent a severe and often fatal problem if diagnosis is delayed. Since many tumors that occur in the mediastinum are undifferentiated and have overlapping histological features, optimal evaluation and diagnosis of mediastinal tumors require an integrated clinical, radiological, and histological approach. The patient demographics in our study showed male-to-female ratio of $3: 2$ that was similar to others..$^{[2,13,14]}$

The mean age in this study was 44.7 years (Range 16-75 years). Variation in the mean age in different studies may be explained by the fact that some studies included infant and pediatric age group patients also in their series. ${ }^{[15-17]}$ Anterior mediastinal 
masses were common in $<30$ years, common causes being thymoma or lymphoma, while in people $>40$ years, mediastinal lymphadenopathy due to metastasis and tuberculosis were common, presenting as middle mediastinal masses.

In this study, 93\% (68) of patients were symptomatic. Other studies have shown a varied incidence. ${ }^{[12,18-22]}$ The common cardinal respiratory symptom with which patients presented was breathlessness $(49.3 \%)$, followed by cough $(42.5 \%)$ and chest pain $(20.5 \%)$. A similar finding was reported in the literature. ${ }^{[17,19,23]}$ In the present study population, cough was associated significantly with middle mediastinal masses. This

Table 3: Final diagnosis and location of mediastinal masses

\begin{tabular}{lc}
\hline Final diagnosis & $\boldsymbol{n}(\%)$ \\
\hline Anterior mediastinal masses & $20(27.4)$ \\
Thymoma & $10(50)$ \\
Neuroendocrine tumor & $4(20)$ \\
Lymphoma & $2(10)$ \\
Mature cystic teratoma & $1(5)$ \\
Retrosternal thyroid carcinoma & $1(5)$ \\
Thymolipoma & $1(5)$ \\
Granulomatous lymphadenitis-Tuberculosis & $1(5)$ \\
Middle mediastinal masses & $42(57.5)$ \\
Granulomatous lymphadenitis - tuberculosis & $14(33.3)$ \\
Granulomatous lymphadenitis - sarcoidosis & $10(23.8)$ \\
Lung cancer & $9(21.4)$ \\
Metastasis & $2(4.8)$ \\
Inconclusive & $7(16.7)$ \\
Posterior mediastinal masses & $8(11.0)$ \\
Solitary fibroid tumor & $1(12.5)$ \\
Poorly differentiated carcinoma & $1(12.5)$ \\
Castleman's disease & $1(12.5)$ \\
Schwannoma & $1(12.5)$ \\
Bronchial cyst & $1(12.5)$ \\
Granulomatous lymphadenitis - sarcoidosis & $1(12.5)$ \\
Tuberculous paraspinal abscess & $1(12.5)$ \\
Inconclusive & $1(12.5)$ \\
Multiple compartment masses & $3(4.1)$ \\
Schwannoma & $1(33.3)$ \\
Lung cancer (small cell carcinoma) & $1(33.3)$ \\
Thymoma & $1(33.3)$ \\
\hline
\end{tabular}

is possibly because $50 \%$ of middle mediastinal masses were associated with a parenchymal lesion or are located closer to larger airways with cough receptors.

Systemic syndrome was seen in eight patients among the total of 73 patients. Five $(6.8 \%)$ of them had myasthenia gravis was the common systemic syndrome and were seen in one-fourth of anterior mediastinal masses (25\%). All these patients were diagnosed as thymoma. Baram and Tayeb ${ }^{[14]}$ reported myasthenia gravis in $27.9 \%$ of anterior mediastinal masses. Myasthenia gravis was present in $45.5 \%$ of patients with thymoma in our study. Thymomas are related to many systemic syndromes, most common being myasthenia gravis. Nearly $5 \%-15 \%$ of patients with myasthenia gravis will be found to have thymoma and $40 \%-45 \%$ of patients with thymoma will present with myasthenia gravis.

The imaging modalities for evaluation of mediastinal masses include chest radiographs, ultrasound, CT scan, magnetic resonance imaging, and nuclear medicine studies. Chest X-rays showed evidence of mediastinal masses in only $34(46.6 \%)$ cases in our study. CT scan imaging of chest showed that the middle mediastinum (57.5\%) was the most common location of mediastinal masses, followed by anterior mediastinal masses $(27.4 \%)$, posterior mediastinal masses (11.0\%), and multiple compartment (4.1\%) cases. In other studies, anterior mediastinal mass was more common than middle mediastinal mass. The newer techniques EBUS and EUS are predominant methods used for evaluation of middle mediastinal mass, especially for mediastinal lymphadenopathy. The availability of the newer techniques EBUS-TBNA and EUS-FNA at our hospital enabled us to evaluate more middle mediastinal masses to obtain confirmatory tissue diagnosis. This might explain the higher incidence of middle mediastinal masses in this study. In many studies, EBUS and EUS were not used in mediastinal masses workup. The incidence of masses in the posterior mediastinum was $11.0 \%$, and comparable with studies by Yadlapalli et al. ${ }^{[24]}(10.4 \%)$ and Aroor et al. ${ }^{[25]}(8.57 \%)$. The shape of mediastinal mass in CT scan was commonly round in $29(39.7 \%)$ patients followed by irregular in $13(17.8 \%)$ patients. Irregular masses were predominantly malignant in nature, and round-shaped masses were benign in nature.

Table 4: Techniques used to obtain specimens and the location of masses, $\boldsymbol{n}(\%)$

\begin{tabular}{|c|c|c|c|c|c|}
\hline \multirow[t]{2}{*}{ Technique } & \multirow[t]{2}{*}{ Anterior $(n=20)$} & \multicolumn{2}{|c|}{ Location } & \multirow[t]{2}{*}{ Multiple $(n=3)$} & \multirow[t]{2}{*}{ Total $(n=73$} \\
\hline & & Middle $(n=42)$ & Posterior $(n=8)$ & & \\
\hline Surgery & $10(50)$ & 0 & $4(50)$ & 0 & $14(19.2)$ \\
\hline EBUS TBNA & 0 & $24(57.1)$ & 0 & 0 & $24(32.9)$ \\
\hline EUS FNA & 0 & $9(21.4)$ & 0 & 0 & $9(12.3)$ \\
\hline EUS biopsy & 0 & $3(7.1)$ & 0 & 0 & $3(4.1)$ \\
\hline CT-guided biopsy & $10(50)$ & $5(11.9)$ & $3(37.5)$ & $3(100)$ & $21(28.8)$ \\
\hline CT-guided FNAC & 0 & 0 & $1(12.5)$ & 0 & $1(1.4)$ \\
\hline VATS biopsy & 0 & $1(2.4)$ & 0 & 0 & $1(1.4)$ \\
\hline
\end{tabular}

EUS FNA: Endoscopic ultrasound-guided fine-needle aspiration, EBUS TBNA: Endobronchial ultrasound-guided transbronchial needle aspiration, VARS: Video-assisted thoracoscopic surgery, FNAC: Fine-needle aspiration cytology, CT: Computed tomography 
Heterogeneous character of masses was more common in this study, and it was predominantly malignant in nature.

Management of mediastinal mass is based on clinical, radiological, and pathological features. Each factor contributes to diagnosis and treatment decision. Definitive tissue diagnosis is often required before initiating therapy. Several techniques are available to approach mediastinal mass to get a tissue diagnosis. Advent of newer techniques has made tissue sampling from these sites easier. The choice of technique depends on multiple factors such as location of mass, clinical stability of the patient, availability, expertise on certain techniques, and affordability.

There are several modalities to obtain tissue samples for cytological or histological diagnosis of mediastinal lesions, and each modality has its own advantages and disadvantages. The choice of these techniques in diagnostic evaluation of mediastinal lesions depends on local availability and expertise. In our study population, techniques used to obtain tissue were EBUS-TBNA in 24 (32.9\%) patients, followed by CT scan-guided transthoracic needle/biopsy and fine-needle aspiration (FNAC) in $22(30.2 \%)$ patients, surgery (sternotomy or thoracotomy) in $14(19.2 \%)$ patients, EUS-FNA/biopsy in $12(16.4 \%)$ patients, and VATS in $1(1.4 \%)$ patient.

Endoscopic biopsies are generally preferred currently in view of fewer complications and ease compared to surgical interventions. The availability of less invasive techniques such as EBUS and EUS techniques at our hospital enabled us to evaluate mediastinal lymphadenopathy in middle mediastinum, which were difficult to approach using image-guided percutaneous techniques. VATS was done in only one patient. Mediastinoscopy was not done in any patient in our study population. In our study, EBUS, EUS, and VATS were used exclusively to approach middle mediastinal masses, particularly for mediastinal lymphadenopathy.

Mediastinal masses displayed a wide histomorphological spectrum in our study. Thirty-three (45.2\%) of malignant and $32(43.8 \%)$ of non-malignant masses were in equal proportions. Dixit et al. reported mediastinal masses as benign in $3.6 \%$, malignant in $63.3 \%$, and nonneoplastic in $23 \%$. ${ }^{[13]}$ Adler et al. ${ }^{[26]}$ and Jereb and Krašsovec ${ }^{[27]}$ reported about $72 \%$ prevalence of malignancy, whereas Karki and Chalise ${ }^{[16]}$ observed only $26 \%$ malignant lesions in a small series of 27 cases. This discordance in the frequency of malignant masses and nonmalignant masses among the previously published studies might be due to variability in age group, study sample size, and techniques used for tissue diagnosis. Among nonmalignant masses, tuberculosis was the common cause, which included tuberculous mediastinal lymphadenopathies in $15(20.5 \%)$ patients and paraspinal abscess in $1(1.4 \%)$ patient, followed by sarcoidosis each in $11(15.1 \%)$. Thymoma was the common malignant mass in $11(15.1 \%)$ patients, followed by lung carcinoma in $10(13.7 \%)$, neuroendocrine tumors in $5.5 \%,{ }^{[4]}$ and lymphoma in $2(2.7 \%)$ in our study group.
Tuberculosis was the common cause of mediastinal lymphadenopathy in $20.5 \%,{ }^{[15]}$ followed by sarcoidosis in $15.1 \%{ }^{[11]}$ and metastasis in $2.7 \%{ }^{\left[{ }^{[2]}\right.}$ Similar studies were done by Dixit et a1. ${ }^{[13]}$ who reported tuberculosis in $20.1 \%$ of cases and Kaur et al. ${ }^{[25]}$ in $15 \%$ of cases and Shaheen et al. ${ }^{[15]}$ in $5 \%$ cases, whereas Adler et al. ${ }^{[26]}$ reported tuberculosis in none of the patients. This gross difference in the occurrence of tuberculosis is probably due to low prevalence of tuberculosis in Western countries compared to developing countries. Mediastinal lymphadenopathy is expected to be the common mediastinal masses because this is caused by high prevalent disease such as tuberculosis, lung cancer metastasis, lymphoma, and sarcoidosis, especially in very high tuberculosis-prevalent country like India. However, mediastinal lymphadenopathies are difficult to biopsy as they are close to major vessels and airways and located commonly in the middle compartment. Hence, mediastinal lymphadenopathy is underrepresented in many studies owing to the difficulty in obtaining a tissue diagnosis. The newer techniques such as EBUS and EUS make approaching such masses with ease and less complications.

Thymoma was reported in $15.1 \%^{[11]}$ of mediastinal masses in our study which was close to $8.3 \%^{[10]}$ in the study by Kaur et al..$^{[23]}$ and Davis et al. ${ }^{[19]}$ showed that thymic masses represented $17 \%$ of all mediastinal lesions, in which thymoma was $14.7 \%$. Similar to other studies, thymoma was predominately located in anterior mediastinum in our study. Lymphoma accounted for $2.7 \%$ of the mediastinal masses in our study and was very less compared to other studies. Davis et al. ${ }^{[19]}$ reported $16 \%$ and Dixit et al. ${ }^{[13]}$ reported $19.4 \%$. Lymphomas are predominately seen in children rather than adults and peripheral lymphadenopathy usually associated with mediastinal mass in many lymphomas which are easily assessable for biopsy to confirm diagnosis. In our study, we had not included children and those who had a biopsy site other than mediastinal mass. These might be the reasons for lymphoma being reported less in our study group.

Schwannoma occurred in 2.7 (2\%) of patients with mediastinal masses in this study and is similar to the study by Aggarwal et al. ${ }^{[28]}(3.4 \%)$. Schwannoma is the most common mediastinal neurogenic tumor, being responsible for $50 \%$ of mediastinal neurogenic tumors in adults. Bronchogenic cyst, thymolipoma, tuberculous paraspinal abscess, mature cystic teratoma, retrosternal goiter (papillary cell carcinoma), solitary fibrous tumor, and Castleman's disease each was reported in $1.4 \%$ of patients in our study. Heterogeneous group of mediastinal masses reported in our study signifies the requirement of accurate diagnosis in mediastinal mass for further management.

\section{ConcLusion}

We conclude that the mediastinum is a complex anatomical region of thorax which is affected by various mediastinal lesions. CT scan is usually the investigation of choice for mediastinal mass for localization and to plan appropriate approaches to reach the mediastinal mass to obtain tissue for 
Sridhar, et al.: Features of mediastinal masses

pathological examination. The newer endoscopic techniques such as EBUS and EUS-guided fine-needle aspiration or biopsy are generally preferred in view of fewer complications and greater ease compared to surgical procedures for mediastinal mass evaluation. Clinical history, anatomical position, and imaging characteristics allow correct diagnosis in many cases when it is combined with histopathology when imaging appearances are similar in many mediastinal tumors. Specific diagnosis is required in mediastinal lesions since numerous malignant and nonmalignant processes occur at this site with a different management plan to facilitate timely treatment.

\section{Financial support and sponsorship}

Nil.

\section{Conflicts of interest}

There are no conflicts of interest.

\section{RefEREnCES}

1. Wychulis AR, Payne WS, Clagett OT, Woolner LB. Surgical treatment of mediastinal tumors: A 40 year experience. J Thorac Cardiovasc Surg 1971;62:379-92.

2. Shabb NS, Fahl M, Shabb B, Haswani P, Zaatari G. Fine-needle aspiration of the mediastinum: A clinical, radiologic, cytologic, and histologic study of 42 cases. Diagn Cytopathol 1998;19:428-36.

3. Wright CD, Mathisen DJ. Mediastinal tumors: Diagnosis and treatment. World J Surg 2001;25:204-9.

4. Roux BI, Kallichurum S, Shama DM. Mediastinal cysts and tumors. Curr ProblSurg 1984;21:6-77.

5. Cohen AJ, Thompson L, Edwards FH, Bellamy RF. Primary cysts and tumors of the mediastinum. Ann Thorac Surg 1991;51:378-84.

6. Shields TW. Primary tumours and cysts of the mediastinum. In: Shields TW, editor. General Thoracic Surgery. Philadelphia, Pa: Lea \& Febiger; 1983. p. 927-54.

7. Fraser RS, Müller NL, Colman N, Paré PD, editors. The mediastinum. In: Fraser and Paré's Diagnosis of Diseases of the Chest. $4^{\text {th }}$ ed. Philadelphia, Pa: Saunders; 1999. p. 196-234.

8. Felson B. Chest Roentgenology. Philadelphia, Pa: Saunders; 1973.

9. Heitzman ER. The Mediastinum. $2^{\text {nd }}$ ed. New York, NY: Springer-Verlag; 1988.

10. Zylak CJ, Pallie W, Jackson R. Correlative anatomy and computed tomography: A module on the mediastinum. Radiographics 1982;2:555-92.

11. Whitten CR, Khan S, Munneke GJ, Grubnic S. A diagnostic approach to mediastinal abnormalities. Radiographics 2007;27:657-71.

12. Sharma P, Jha V, Kumar N, Kumar R, Mandal A. Clinicopathological analysis of mediastinal masses: A mixed bag of non-neoplastic and neoplastic etiologles. Turk Patoloji Derg 2017;33:37-46.

13. Dixit R, Shah NS, Goyal M, Patil CB, Panjabi M, Gupta RC, et al. Diagnostic evaluation of mediastinal lesions: Analysis of 144 cases. Lung India 2017;34:341-8.

14. Baram A, Tayeb ZA. Mediastinal Masses: Retrospective single center based study. J Cancer Sci Ther 2016;8:252-6.

15. Shaheen MZ, Sardar K, Murtaza HG, Safdar SS, Hafeez A, Mushtaq MA. CT guided trans-thoracic fine needle aspiration/biopsy of Mediastinal and Hilar Mass Lesions: An experience of Pulmonology Department at a tertiary care teaching hospital. Pakistan J Chest Med 2010;16:6-8.

16. Karki S, Chalise S. Analysis of mediastinal lesions: A study of 27 cases. J Pathol Nepal 2011;1:114-7.

17. Bagheri R, Afghani R, Ziaollah Haghi S, Fattahi Masoum SH, Zarehparvar Moghaddam S, Akglaghi S. Evaluation of 95 cases with Mediastinal tumors. J Cardio Thoracic Med 2015;3:249-53.

18. Dubashi B, Cyriac S, Tenali SG. Clinicopathological analysis and outcome of primary mediastinal malignancies A report of 91 cases from a single institute. Ann Thorac Med 2009;4:140-2.

19. Davis RD Jr., Oldham HN Jr., Sabiston DC Jr. Primary cysts and neoplasms of the mediastinum: Recent changes in clinical presentation, methods of diagnosis, management, and results. Ann Thorac Surg 1987;44:229-37.

20. Duwe BV, Sterman DH, Musani AI. Tumors of the mediastinum. Chest 2005;128:2893-909.

21. Vaziri M, Pazooki A, Zahedi-Shoolami L. Mediastinal masses: Review of 105 cases. Acta Med Iranica 2009;47:297-300.

22. Adegboye VO, Ogunseyinde AO, Obajimi MO, Ogunbiyi O, Brimmo AI, Adebo OA. Presentation of primary mediastinal masses in Ibadan. East Afr Med J 2003;80:484-7.

23. Kaur H, Tiwari P, Dugg P, Ghuman J, Shivhare P, Mehmi RS. Computed tomographic evaluation of mediastinal masses/lesions with contrast enhancement and correlation with pathological diagnosis-a study of 120 cases. J Biomed Graphics Computing 2014;4:28-35.

24. Yadlapalli CD, Gumdal V, Punia A, Mishra R, Chitalkar PG, Jain A. Spectrum of malignant mediastenal masses at a tertiary care centre in central India. International J Res Med Sci 2018;6:2343-7.

25. Aroor AR, Rama Prakasha S, Seshadri S, Teerthanath S, Raghuraj U. A study of clinical characteristics of mediastinal mass. J Clin Diagn Res 2014;8:77-80.

26. Adler OB, Rosenberger A, Peleg H. Fine-needle aspiration biopsy of mediastinal masses: Evaluation of 136 experiences. AJR Am J Roentgenol 1983;140:893-6.

27. Jereb M, Us-Krasovec M. Transthoracic needle biopsy of mediastinal and hilar lesions. Cancer 1977;40:1354-7.

28. Aggarwal R, Rao S, Chopra P, Bhalla S, Vijay CL, Asaf BB, et al. Morphological spectrum of mediastinal lesions with special emphasis on evaluation of needle biopsy: An experience from a tertiary care hospital. Indian J Med Res 2016;144:544-51. 